ANNALES

POLONICI MATHEMATICI

$84.1(2004)$

\title{
Averaging techniques and oscillation of quasilinear elliptic equations
}

by Zhi-Ting Xu, BaO-Guo JiA and ShaO-YuAn Xu (Guangzhou)

Abstract. By using averaging techniques, some oscillation criteria for quasilinear elliptic differential equations of second order

$$
\sum_{i, j=1}^{N} D_{i}\left[A_{i j}(x)|D y|^{p-2} D_{j} y\right]+p(x) f(y)=0
$$

are obtained. These results extend and generalize the criteria for linear differential equations due to Kamenev, Philos and Wong.

1. Introduction. We consider quasilinear elliptic differential equations of second order

$$
\sum_{i, j=1}^{N} D_{i}\left[A_{i j}(x)|D y|^{p-2} D_{j} y\right]+p(x) f(y)=0
$$

in $\Omega(a)$, where $p>1, x=\left(x_{1}, \ldots, x_{N}\right) \in \mathbb{R}^{N}, N \geq 2, D y=\left(D_{1} y, \ldots\right.$, $\left.D_{N} y\right), D_{i} y=\partial y / \partial x_{i}$ for $i=1, \ldots, N,|x|=\left[\sum_{i=1}^{N} x_{i}^{2}\right]^{1 / 2}$, and $\Omega(a)=$ $\left\{x \in \mathbb{R}^{N}:|x| \geq a\right\}$ for some $a>0$.

Throughout this paper we shall assume that the following conditions hold.

$\left(\mathrm{A}_{1}\right) \quad A=\left(A_{i j}(x)\right)_{N \times N}$ is a real symmetric positive definite matrix function with $A_{i j} \in C_{\mathrm{loc}}^{1+\mu}(\Omega(a), \mathbb{R}), i, j=1, \ldots, N, 0<\mu<1$. Denote by $\lambda_{\max }(x)$ the largest eigenvalue of the matrix $A$. There is a function $\lambda \in C\left([a, \infty), \mathbb{R}^{+}\right)$such that

$$
\min _{|x|=r} \frac{|A|^{(p-2) /(p-1)}}{\lambda_{\max }(x)} \geq \lambda(r), \quad r>a
$$

where $|A|$ denotes the norm of $A$, i.e., $|A|=\left[\sum_{i, j=1}^{N} A_{i j}^{2}(x)\right]^{1 / 2}$.

2000 Mathematics Subject Classification: 35B05, 35J60, 34C10.

Key words and phrases: oscillation, quasilinear elliptic equation, second order, averaging technique. 
$\left(\mathrm{A}_{2}\right) \quad p \in C_{\mathrm{loc}}^{\mu}(\Omega(a), \mathbb{R}), 0<\mu<1$

$\left(\mathrm{A}_{3}\right) \quad f \in C^{1}(\mathbb{R}, \mathbb{R}), y f(y)>0$, and $f^{\prime}(y) /|f(y)|^{(p-2) /(p-1)} \geq k>0$ for $y \neq 0$.

In what follows, a solution of (1.1) is every function of class $C_{\mathrm{loc}}^{2+\mu}(\Omega(a), \mathbb{R})$, $0<\mu<1$, which satisfies (1.1) almost everywhere on $\Omega(a)$. We consider only nontrivial solutions of (1.1) which are defined for all large $|x|$ (cf. [1]). The oscillation is considered in the usual sense, i.e., a solution $y(x)$ of $(1.1)$ is said to be oscillatory if it has arbitrarily large zeros, i.e., the set $\left\{x \in \mathbb{R}^{N}\right.$ : $y(x)=0\}$ is unbounded. Equation (1.1) is said to be oscillatory if every solution (if any exists) is oscillatory. Conversely, (1.1) is nonoscillatory if there exists a solution which is not oscillatory.

If $p=2$, then equation (1.1) reduces to the semilinear elliptic equation

$$
\sum_{i, j=1}^{N} D_{i}\left[A_{i j}(x) D_{j} y\right]+p(x) f(y)=0,
$$

and if $A(x)=I$ (identity matrix), $f(y)=|y|^{p-2} y$, then (1.1) reduces to the PDE with $p$-Laplacian

$$
\operatorname{div}\left(|D y|^{p-2} D y\right)+p(x)|y|^{p-2} y=0 .
$$

Many results guaranteeing that the solutions of (1.2) are oscillatory can be found in the literature (see, for example, $[3,5,9-12])$. The oscillation criteria of Kamenev [2], Philos [4], Wintner [7] and others for the second order linear differential equation

$$
\left(r(t) y^{\prime}(t)\right)^{\prime}+p(t) y(t)=0, \quad p \in C\left(\left[t_{0}, \infty\right), \mathbb{R}^{+}\right),
$$

have been extended to (1.2). Recently, H. Usami [6] established a Wintnertype oscillation theorem [7] for (1.3). However, to the best of our knowledge, equation (1.1) in its general form does not seem to have been the object of systematic investigation. Motivated by this fact, we try to develop oscillation theory for (1.1). In this paper, by using averaging technique [2] and general means $[4,8]$, we establish some oscillation criteria for (1.1), thereby generalizing results of Kamenev [2], Philos [4] and Wong [8] to (1.1). Our approach is somewhat different from that of the previous authors. We believe that our approach is simpler and also provides a more unified account of Kamenev-type oscillation theorems.

2. Main results. First of all, we introduce the general means $[4,8]$ and present some properties which will be used in the proof of our results.

Let $D=\{(r, s): r \geq s \geq a\}$ and $D_{0}=\{(r, s): r>s \geq a\}$. We will say that the function $H \in C(D, \mathbb{R})$ belongs to the class $\Im$ (written $H \in \Im$ ) if

$$
H(r, r)=0 \text { for } r \geq a, H(r, s)>0 \text { on } D_{0} ;
$$


$\left(\mathrm{H}_{2}\right) \quad H$ has a continuous and nonpositive partial derivative in $D_{0}$ with respect to the second variable;

$\left(\mathrm{H}_{3}\right) \quad$ there exist $\varrho \in C^{1}\left([a, \infty), \mathbb{R}^{+}\right)$and $h \in C(D, \mathbb{R})$ such that

$$
\frac{\partial}{\partial s}[H(r, s) \varrho(s)]=-H(r, s) h(r, s), \quad(r, s) \in D_{0} .
$$

Let $\varrho \in C^{1}\left([a, \infty), \mathbb{R}^{+}\right)$and $H \in \Im$. We consider the integral operator

$$
\Theta_{b}^{\varrho}(\phi ; r)=\int_{b}^{r} H(r, s) \phi(s) \varrho(s) d s, \quad r \geq b \geq a,
$$

where $\phi \in C([a, \infty), \mathbb{R})$ (see [8]). It is easily seen that

$$
\begin{aligned}
& \Theta_{b}^{\varrho}\left(\alpha_{1} h_{1}+\alpha_{2} h_{2} ; r\right)=\alpha_{1} \Theta_{b}^{\varrho}\left(h_{1}, r\right)+\alpha_{2} \Theta_{b}^{\varrho}\left(h_{2}, r\right), \\
& \Theta_{b}^{\varrho}\left(h_{3}, r\right) \geq 0 \quad \text { whenever } h_{3} \geq 0, \\
& \Theta_{b}^{\varrho}\left(h_{4}^{\prime} ; r\right)=-H(r, b) h_{4}(b) \varrho(b)+\Theta_{b}^{\varrho}\left(\varrho^{-1} h_{4} h ; r\right) .
\end{aligned}
$$

Here $h_{i} \in C([a, \infty), \mathbb{R})$ and $\alpha_{1}, \alpha_{2}$ are real numbers.

For each $\xi \in C\left([a, \infty), \mathbb{R}^{+}\right)$with $\int_{a}^{\infty}(1 / \xi(\tau)) d \tau=\infty$, define the kernel function

$$
H(r, s)=\left(\int_{s}^{r} \frac{d u}{\xi(u)}\right)^{m}, \quad m>p-1 .
$$

For example, an important particular case is $\xi(\tau)=\tau^{n}$, where $n \leq 1$ is real. When $\xi(\tau)=1$ we have $H(r, s)=(r-s)^{m}$, and when $\xi(\tau)=\tau$ we have $H(r, s)=(\ln r / \ln s)^{m}$. It is easily verified that the kernel function (2.5) satisfies $\left(\mathrm{H}_{1}\right)-\left(\mathrm{H}_{3}\right)$.

Theorem 2.1. Suppose that there exist $\phi \in C^{1}\left([a, \infty), \mathbb{R}^{+}\right)$and $H, h \in$ $C(D, \mathbb{R})$ such that $H \in \Im$ and

$$
\limsup _{r \rightarrow \infty} \frac{1}{H(r, a)} \Theta_{a}^{\varrho}\left(\varphi-p^{-p} g^{1-p}|h|^{p} ; r\right)=\infty,
$$

where

$$
\begin{aligned}
& g(r)=\frac{k p}{p^{2}-1} \lambda(r)[\varrho(r)]^{q}\left[\omega_{N} r^{N-1} \phi(r)\right]^{1 /(1-p)}, \\
& \varphi(r)=\phi(r) \int_{S_{r}} p(x) d \sigma-\frac{1}{p}[g(r)]^{1-p}[\varrho(r)]^{p}\left|\frac{\phi^{\prime}(r)}{\phi(r)}\right|^{p},
\end{aligned}
$$

$q$ is the conjugate number to $p$, i.e., $q=p /(p-1), S_{r}=\left\{x \in \mathbb{R}^{N}:|x|=r\right\}$, $r>0, \sigma$ denotes the measure on $S_{r}$ and $\omega_{N}$ denotes the surface of the unit sphere in $\mathbb{R}^{N}$, i.e, $\omega_{N}=2 \pi^{N / 2} / \Gamma(N / 2)$. Then equation (1.1) is oscillatory.

Proof. Suppose $y(x)$ is a nonoscillatory solution of (1.1). Without loss of generality we assume that $y(x)>0$ for $|x|>a$. Hence, the $N$-dimensional 
vector Riccati operator

$$
W(x)=\frac{1}{f(y)} A(x)|D y|^{p-2} D y
$$

exists on $\Omega(a)$. By differentiating $W(x)$ and making use of (1.1), we see that, for all $x \in \Omega(a), W(x)$ satisfies the Riccati equation

$$
\operatorname{div} W(x)=-p(x)-f^{\prime}(y)|D y|^{2-p}\left(W^{T} A^{-1} W\right)(x) .
$$

Note that

$$
|W(x)| \leq \frac{1}{|f(y)|}|A(x)||D y|^{p-1}
$$

and

$$
\left(W^{T} A^{-1} W\right)(x) \geq \lambda_{\max }^{-1}(x)|W(x)|^{2} .
$$

Then (2.8) implies that

$$
\operatorname{div} W(x) \leq-p(x)-\frac{f^{\prime}(y)}{|f(y)|^{(p-2) /(p-1)}} \frac{|A(x)|^{(p-2) /(p-1)}}{\lambda_{\max }(x)}|W(x)|^{q} .
$$

Put

$$
Z(r)=\phi(r) \int_{S_{r}} W(x) \cdot \nu(x) d \sigma
$$

where $\nu(x)=x /|x|,|x| \neq 0$, denotes the outward unit normal to $S_{r}, r=|x|$. By means of the Green formula and (2.9), we obtain

$$
\begin{aligned}
Z^{\prime}(r) & =\frac{\phi^{\prime}(r)}{\phi(r)} Z(r)+\phi(r) \int_{S_{r}} \operatorname{div} W(x) d \sigma \\
& \leq \frac{\phi^{\prime}(r)}{\phi(r)} Z(r)-\phi(r)\left[\int_{S_{r}} p(x) d \sigma+k \lambda(r) \int_{S_{r}}|W(x)|^{q} d \sigma\right] .
\end{aligned}
$$

The Hölder inequality yields

$$
\begin{aligned}
|Z(r)| & \leq \phi(r)\left[\int_{S_{r}}|\nu(x)|^{p} d \sigma\right]^{1 / p}\left[\int_{S_{r}}|W(x)|^{q} d \sigma\right]^{1 / q} \\
& \leq \phi(r)\left[\omega_{N} r^{N-1}\right]^{1 / p}\left[\int_{S_{r}}|W(x)|^{q} d \sigma\right]^{1 / q}
\end{aligned}
$$

which is equivalent to

$$
\int_{S_{r}}|W(x)|^{q} d \sigma \geq[\phi(r)]^{-q}\left[\omega_{N} r^{N-1}\right]^{1 /(1-p)}|Z(r)|^{q} .
$$

Thus, (2.10) gives

$$
Z^{\prime}(r) \leq-\phi(r) \int_{S_{r}} p(x) d \sigma+\frac{\phi^{\prime}(r)}{\phi(r)} Z(r)-\frac{p^{2}-1}{p} g(r)[\varrho(r)]^{-q}|Z(r)|^{q} .
$$


The Young inequality implies that

$$
\left|\frac{\phi^{\prime}(r)}{\phi(r)} Z(r)\right| \leq \frac{1}{q} g(r)[\varrho(r)]^{-q}|Z(r)|^{q}+\frac{1}{p}[g(r)]^{1-p}[\varrho(r)]^{p}\left|\frac{\phi^{\prime}(r)}{\varrho(r)}\right|^{p} .
$$

Combining this inequality with (2.11) gives

$$
Z^{\prime}(r) \leq-\varphi(r)-(p-1) g(r)[\varrho(r)]^{-q}|Z(r)|^{q} .
$$

Applying the operator $\Theta_{b}^{\varrho}, b \geq a$, to (2.12) and using (2.4), we obtain

$$
\begin{aligned}
\Theta_{b}^{\varrho}(\varphi ; r) \leq & H(r, b) \varrho(b) Z(b)+\Theta_{b}^{\varrho}\left(\varrho^{-1}|h||Z| ; r\right) \\
& -(p-1) \Theta_{b}^{\varrho}\left(g \varrho^{-q}|Z|^{q} ; r\right) .
\end{aligned}
$$

In view of the Young inequality,

$$
\varrho^{-1}|h||Z| \leq(p-1) g \varrho^{-q}|Z|^{q}+p^{-p} g^{1-p}|h|^{p} .
$$

Substituting the above inequality into (2.13), we get

$$
\Theta_{b}^{\varrho}(\varphi ; r) \leq H(r, b) \varrho(b) Z(b)+p^{-p} \Theta_{b}^{\varrho}\left(g^{1-p}|h|^{p} ; r\right) .
$$

Set $b=a$ and divide (2.14) through by $H(r, a)$, then take limsup as $r \rightarrow \infty$. Condition (2.6) gives the desired contradiction.

As an immediate consequence of Theorem 2.1, we obtain the following corollary.

COROLlary 2.1. Let the assumptions of Theorem 2.1 be satisfied except that condition (2.6) is replaced by

$$
\limsup _{r \rightarrow \infty} \frac{1}{H(r, a)} \Theta_{a}^{\varrho}(\varphi ; r)=\infty,
$$

and

$$
\limsup _{r \rightarrow \infty} \frac{1}{H(r, a)} \Theta_{a}^{\varrho}\left(g^{1-p}|h|^{p} ; r\right)<\infty .
$$

Then equation (1.1) is oscillatory.

Note that condition (2.15) in Theorem 2.1 is necessary. In the remainder of this paper, we do not require this condition, but will have some other conditions instead. The following result provides an essentially new oscillation theorem for (1.1).

Theorem 2.2. Let $H$ and $h$ be as in Theorem 2.1. Suppose that there exist $\phi_{1}, \phi_{2} \in C([a, \infty), \mathbb{R})$ such that for all $b \geq a$,

$$
\begin{gathered}
\limsup _{r \rightarrow \infty} \frac{1}{H(r, b)} \Theta_{b}^{\varrho}(\varphi ; r) \geq \phi_{1}(b), \\
\limsup _{r \rightarrow \infty} \frac{1}{H(r, b)} \Theta_{b}^{\varrho}\left(g^{1-p}|h|^{p} ; r\right) \leq \phi_{2}(b),
\end{gathered}
$$


where $\phi_{1}$ and $\phi_{2}$ satisfy

$$
\liminf _{r \rightarrow \infty} \frac{1}{H(r, a)} \Theta_{a}^{\varrho}\left(g \varrho^{-2 q}\left(\varphi_{1}-p^{-p} \phi_{2}\right)_{+}^{q} ; r\right)=\infty,
$$

where $\phi_{+}=\max \{0, \phi\}$. Then equation (1.1) is oscillatory.

Proof. We proceed as in the proof of Theorem 2.1 to get (2.13) and (2.14). Then, by (2.14), we have, for all $r>b \geq a$,

$$
\frac{1}{H(r, b)} \Theta_{b}^{\varrho}(\varphi ; r)-\frac{1}{p^{p} H(r, b)} \Theta_{b}^{\varrho}\left(g^{1-p}|h|^{p} ; r\right) \leq \varrho(b) Z(b) .
$$

Taking limsup in the above inequality as $r \rightarrow \infty$ and applying (2.17) and (2.18), we obtain

$$
\phi_{1}(b)-p^{-p} \phi_{2}(b) \leq \varrho(b) Z(b),
$$

from which it follows that

$$
\frac{1}{H(r, b)} \Theta_{b}^{\varrho}\left(g \varrho^{-2 q}\left(\phi_{1}-p^{-p} \phi_{2}\right)_{+}^{q} ; r\right) \leq \frac{1}{H(r, b)} \Theta_{b}^{\varrho}\left(g \varrho^{-q}|Z|^{q} ; r\right) .
$$

On the other hand, by (2.13),

$$
\begin{aligned}
\frac{p-1}{H(r, b)} \Theta_{b}^{\varrho}\left(g \varrho^{-q}|Z|^{q} ; r\right)-\frac{1}{H(r, b)} \Theta_{b}^{\varrho}\left(\varrho^{-1}|h||Z| ; r\right) & \\
& \leq \varrho(b) Z(b)-\frac{1}{H(r, b)} \Theta_{b}^{\varrho}(\varphi ; r) .
\end{aligned}
$$

Thus, by (2.17),

$$
\begin{array}{r}
\liminf _{r \rightarrow \infty}\left\{\frac{p-1}{H(r, b)} \Theta_{b}^{\varrho}\left(g \varrho^{-q}|Z|^{q} ; r\right)-\frac{1}{H(r, b)} \Theta_{b}^{\varrho}\left(\varrho^{-1}|h||Z| ; r\right)\right\} \\
\leq \varrho(b) Z(b)-\phi_{1}(b) \leq C_{0}
\end{array}
$$

where $C_{0}$ is a constant.

Now, we claim that

$$
\liminf _{r \rightarrow \infty} \frac{1}{H(r, b)} \Theta_{b}^{\varrho}\left(g \varrho^{-q}|Z|^{q} ; r\right)<\infty .
$$

If (2.22) does not hold, then there exists a sequence $\left\{r_{j}\right\}_{j=1}^{\infty} \subset[a, \infty)$ with $\lim _{j \rightarrow \infty} r_{j}=\infty$ such that

$$
\lim _{j \rightarrow \infty} \frac{1}{H\left(r_{j}, b\right)} \Theta_{b}^{\varrho}\left(g \varrho^{-q}|Z|^{q} ; r_{j}\right)=\infty .
$$

Hence, by (2.21), for $j$ large enough, we have

$$
\frac{p-1}{H\left(r_{j}, b\right)} \Theta_{b}^{\varrho}\left(g \varrho^{-q}|Z|^{q} ; r_{j}\right)-\frac{1}{H\left(r_{j} ; b\right)} \Theta_{b}^{\varrho}\left(\varrho^{-1}|h||Z| ; r_{j}\right) \leq C_{0}+1 .
$$


This and (2.23) give, for $j$ large enough,

$$
\frac{\Theta_{b}^{\varrho}\left(\varrho^{-1}|h||Z| ; r_{j}\right)}{\Theta_{b}^{\varrho}\left(g \varrho^{-q}|Z|^{q} ; r_{j}\right)}-(p-1) \geq-\frac{1}{2}(p-1),
$$

that is,

$$
\Theta_{b}^{\varrho}\left(\varrho^{-1}|h||Z| ; r_{j}\right) \geq \frac{p-1}{2} \Theta_{b}^{\varrho}\left(g \varrho^{-q}|Z|^{q} ; r_{j}\right) \quad \text { for all large } j .
$$

By the Hölder inequality,

$$
\Theta_{b}^{\varrho}\left(\varrho^{-1} h|Z| ; r_{j}\right) \leq\left[\Theta_{b}^{\varrho}\left(g \varrho^{-q}|Z|^{q} ; r_{j}\right)\right]^{1 / q}\left[\Theta_{b}^{\varrho}\left(g^{1-p}|h|^{p} ; r_{j}\right)\right]^{1 / p} .
$$

From (2.24) and (2.25), we obtain

$$
\left(\frac{p-1}{2}\right)^{p} \Theta_{b}^{\varrho}\left(g \varrho^{-q}|Z|^{q} ; r_{j}\right) \leq \Theta_{b}^{\varrho}\left(g^{1-p}|h|^{p} ; r_{j}\right) .
$$

By (2.18), the right-hand side of (2.26) is bounded, which contradicts (2.23). Therefore, (2.22) holds. Hence, by (2.20),

$$
\begin{aligned}
\liminf _{r \rightarrow \infty} \frac{1}{H(r, b)} \Theta_{b}^{\varrho}\left(g \varrho ^ { - 2 q } \left(\phi_{1}-\right.\right. & \left.\left.p^{-p} \phi_{2}\right)_{+}^{q} ; r\right) \\
& \leq \liminf _{r \rightarrow \infty} \frac{1}{H(r, b)} \Theta_{b}^{\varrho}\left(g \varrho^{-q}|Z|^{q} ; r\right)<\infty,
\end{aligned}
$$

which contradicts (2.19).

3. Corollaries and examples. As Theorems 2.1 and 2.2 are rather general, it is convenient for applications to derive a number of oscillation criteria with the appropriate choice of the functions $H, \phi$ and $\varrho$.

Let us first introduce an assumption:

$\left(\mathrm{A}_{4}\right) \quad$ There exist constants $C>0$ and $\beta$ such that

$$
\lambda(r) \geq C r^{\beta q}, \quad r \geq a .
$$

Now, we establish Kamenev-type oscillation criteria for (1.1). Let

$$
H(r, s)=(r-s)^{\alpha}, \quad \varrho(s)=s^{-\alpha}, \quad \phi(r)=r^{1-N},
$$

where $\alpha>1$ is a constant. Then it is easy to see that

$$
h(r, s)=\alpha(r-s)^{-1} s^{-\alpha-1} r, \quad g(r) \geq C_{1} r^{(\beta-\alpha) q},
$$

and

$$
\varphi(r) \geq \omega_{N} \bar{p}(r)-C_{2} r^{-(\beta+1) p},
$$

where

$\bar{p}(r)=\frac{1}{\omega_{N} r^{N-1}} \int_{S_{r}} p(x) d \sigma, \quad C_{1}=\frac{k p C}{p^{2}-1} \omega_{N}^{1 /(1-p)}, \quad C_{2}=\frac{1}{p} C_{1}^{1-p}(N-1)^{p}$. 
A direct computation yields

$$
\Theta_{a}^{\varrho}\left(g^{1-p}|h|^{p} ; r\right) \leq C_{1}^{1-p} \alpha^{p} r^{p} \int_{a}^{r}(r-s)^{\alpha-p} s^{-(\beta+1) p-\alpha} d s
$$

and

$$
\Theta_{a}^{\varrho}(\varphi ; r) \geq \int_{a}^{r}(r-s)^{\alpha}\left[\omega_{N} \bar{p}(s) s^{-\alpha}-C_{2} s^{-(\beta+1) p-\alpha}\right] d s .
$$

Hence, by Theorem 2.1, we have

Corollary 3.1 Let $\left(\mathrm{A}_{4}\right)$ hold. If there exists a constant $\alpha>1$ with $(\beta+1) p+\alpha>1$ such that

$$
\limsup _{r \rightarrow \infty} r^{-\alpha} \int_{a}^{r}(r-s)^{\alpha} \bar{p}(s) s^{-\alpha} d s=\infty
$$

then equation (1.1) is oscillatory.

Corollary 3.2. Suppose that there exists $\phi \in C^{1}([a, \infty), \mathbb{R})$ such that

$$
\limsup _{r \rightarrow \infty} G^{-\alpha}(r) \int_{a}^{r}[G(r)-G(s)]^{\alpha} \varphi_{1}(s) d s=\infty \quad \text { for some } \alpha>p-1
$$

where

$$
\begin{aligned}
& g_{1}(r)=\frac{k p}{p^{2}-1} \lambda(r)\left[\omega_{N} r^{N-1} \phi(r)\right]^{1 /(1-p)}, \quad G(r)=\int_{a}^{r} g_{1}(s) d s \\
& \varphi_{1}(r)=\phi(r) \int_{S_{r}} p(x) d \sigma-\frac{1}{p}\left[g_{1}(r)\right]^{1-p}\left|\frac{\phi^{\prime}(r)}{\phi(r)}\right|^{p} .
\end{aligned}
$$

Then equation (1.1) is oscillatory.

Proof. Let

$$
H(r, s)=[G(r)-G(s)]^{\alpha}, \quad \varrho(r)=1
$$

Then

$$
h(r, s)=\alpha[G(r)-G(s)]^{-1} g_{1}(s)
$$

and

$$
\int_{a}^{r} H(r, s) g_{1}^{1-p}(s)|h(r, s)|^{p} d s=\frac{\alpha^{p}}{\alpha-p+1}[G(r)]^{\alpha-p+1} .
$$

This implies

$$
\begin{aligned}
& \limsup _{r \rightarrow \infty} \frac{1}{H(r, a)} \Theta_{a}^{\varrho}\left(\varphi-p^{-p} g^{1-p}|h|^{p} ; r\right) \\
& \quad=\limsup _{r \rightarrow \infty}\left\{G^{-\alpha}(r) \int_{a}^{r}[G(r)-G(s)]^{\alpha} \varphi_{1}(s) d s-\frac{p^{-p} \alpha^{p}}{\alpha-p+1}[G(r)]^{1-p}\right\}=\infty,
\end{aligned}
$$

and it follows from Theorem 2.1 that (1.1) is oscillatory. 
REMARK 3.1. Corollary 3.2 improves Theorem 4 for equation (1.2) in [3] and Theorem 4 for equation (1.3) in [6].

By Theorem 2.2, we have

Corollary 3.3. Assume that $\lim _{r \rightarrow \infty} G(r)=\infty$. Suppose that there exist $\phi \in C^{1}\left([a, \infty), \mathbb{R}^{+}\right)$and $\psi \in C([a, \infty), \mathbb{R})$ such that for all $b>a$,

$$
\begin{gathered}
\limsup _{r \rightarrow \infty} G^{-\alpha}(r) \int_{b}^{r}[G(r)-G(s)]^{\alpha} \varphi_{1}(s) d s \geq \psi(b), \\
\liminf _{r \rightarrow \infty} G^{-\alpha}(r) \int_{b}^{r} g_{1}(s)[G(r)-G(s)]^{\alpha}[\psi(s)]_{+}^{q} d s=\infty,
\end{gathered}
$$

for some $\alpha>p-1$. Then equation (1.1) is oscillatory.

Example 3.1. Consider

$$
\sum_{i, j=1}^{N} D_{i}\left[A_{i j}(x)|D y|^{p-2} D_{j} y\right]+\frac{1+k \sin |x|}{|x|^{\nu}}=0,
$$

where $x \in \Omega(1), A(x)=\operatorname{diag}(|x|, \ldots,|x|), p>1, \bar{p}(r)=(1+k \sin r) / r^{\nu}$, $\lambda(r)=N^{(p-2) / 2(1-p)} r^{1 /(1-p)}$. By Corollary 3.1, if there exists a constant $\alpha>1$ with $\nu+\alpha \leq 1$, then (3.6) is oscillatory.

EXAMPle 3.2. Consider the equation

$$
\operatorname{div}\left(|D y|^{p-2} D y\right)+\left(|x|^{\nu} \cos |x|\right)|y|^{p-2} y=0,
$$

where $x \in \Omega(1), \lambda(r)=N^{(p-2) / 2(p-1)}, 3>p>1, \nu \leq 1$. Let $\varrho(r)=1$, $\phi(r)=r^{1-N}$. A direct computation implies

$$
g_{1}(r)=C_{3}, \quad \varphi_{1}(r)=\omega_{N} r^{\nu} \cos r-\frac{C_{4}}{r^{p}},
$$

where

$$
\begin{aligned}
& C_{3}=\frac{p}{p^{2}-1} N^{(p-2) / 2(p-1)} \omega_{N}^{1 /(1-p)}, \\
& C_{4}=N^{(2-p) / 2}(N-1)^{p} \frac{\omega_{N}}{p}\left(\frac{p}{p^{2}-1}\right)^{1-p} .
\end{aligned}
$$

In Corollary 3.3, let $\alpha=2$. We have

$$
\limsup _{r \rightarrow \infty} \frac{1}{G^{2}(r)} \int_{b}^{r}[G(r)-G(s)]^{2} \varphi_{1}(s) d s \geq-r^{\nu} \sin b-\epsilon .
$$

Now, set $\psi(b)=-b^{\nu} \sin b-\epsilon$ and consider an integer $J$ such that $2 J \pi+$ $5 \pi / 4 \geq \max \left\{a,(1+\sqrt{2} \epsilon)^{1 / \nu}\right\}$. Then, for all integers $n>J$, we have

$$
\psi(b) \geq 1 / \sqrt{2} \text { for every } b \in[2 n \pi+5 \nu / 4,2 n \pi+7 \pi / 4],
$$

which implies that 


$$
\begin{aligned}
\liminf _{r \rightarrow \infty} \frac{1}{G^{2}(r)} \int_{a}^{r} g_{1}(s)[G(r)-G(s)]^{2}[\psi(s)]_{+}^{q} d s & \\
& \geq C_{3} \sum_{n=J}^{\infty}(\sqrt{2})^{-q} \int_{2 n \pi+5 \pi / 4}^{2 n \pi+7 \pi / 4} d s=\infty
\end{aligned}
$$

Then, by Corollary 3.3, equation (3.7) is oscillatory.

Acknowledgments. We would like to express our gratitude to the anonymous referee for careful reading and helpful suggestions which led to an improvement of the original manuscript.

\section{References}

[1] J. I. Díaz, Nonlinear Partial Differential Equations and Free Boundaries, Vol. I, Elliptic Equations, Pitman, London, 1985.

[2] I. V. Kamenev, An integral criterion for oscillation of linear differential equations, Mat. Zametki 23 (1978), 249-251 (in Russian).

[3] E. S. Noussair and C. A. Swanson, Oscillation of semilinear elliptic inequalities by Riccati transformations, Canad. J. Math. 32 (1980), 908-923.

[4] Ch. G. Philos, Oscillation theorems for linear differential equation of second order, Arch. Math (Basel) 53 (1989), 482-492.

[5] C. A. Swanson, Semilinear second order elliptic oscillation, Canad. Math. Bull. 22 (1979), 139-157.

[6] H. Usami, Some oscillation theorems for a class of quasilinear elliptic equations, Ann. Mat. Pura Appl. 175 (1998), 277-283.

[7] A. Wintner, A criterion of oscillatory stability, Quart. J. Appl. Math. 7 (1949), 115-117.

[8] J. S. W. Wong, On Kamenev-type oscillation theorems for second-order differential equations with damping, J. Math. Anal. Appl. 258 (2001), 244-257.

[9] Z. T. Xu, Oscillation of solutions to second order elliptic partial differential equations with a "weakly integrally small" coefficient, J. Systems Sci. Math. Sci. 18 (1998), 478-484 (in Chinese).

[10] - Riccati techniques and oscillation of semilinear elliptic equations, Chinese Ann. Math. Ser. A 24 (2003), 565-574 (in Chinese).

[11] Z. T. Xu, D. K. Ma and B. G. Jia, Oscillation theorems for elliptic equations of second order, Acta Math. Sci. Ser. A 24 (2004), 144-151 (in Chinese).

[12] B. G. Zhang, T. Zhao and B. S. Lalli, Oscillation criteria for nonlinear second order elliptic differential equations, Chinese Ann. Math. Ser. B 17 (1996), 89-102.

Department of Mathematics

South China Normal University

Guangzhou 510631, P.R. China

E-mail: xztxhyyj@pub.guangzhou.gd.cn
Mathematics and Scientific Computer College Zhongshan University Guangzhou 510275, P.R. China 